\title{
Servicios ecosistémicos de regulación en playas del partido de General Alvarado, Buenos Aires, Argentina
}

\author{
Regulating ecosystem services of beaches in General \\ Alvarado Municipality, Buenos Aires, Argentina
}

\author{
Alejandra Merlotto1, Eleonora M. Verón y Germán R. Bértola ${ }^{3}$
}

\begin{abstract}
RESUMEN
La provisión de servicios ecosistémicos ha sido alterada en las áreas litorales de todo el mundo debido al intenso proceso de urbanización de las áreas costeras. Los servicios de regulación de las playas han sido modificados especialmente en áreas en las cuales se desarrolla la práctica turística. El objetivo del presente trabajo fue evaluar el estado de los servicios de regulación morfo-sedimentaria y a perturbaciones naturales del ecosistema costero en las ciudades de Miramar y Mar del Sur (Argentina). Se seleccionaron indicadores naturales y antrópicos y se evaluaron cinco playas con características geomorfológicas diferentes y con diversos grados de intervención antrópica. La provisión de los servicios resultó buena en las playas con menor intervención antrópica, mientras que en las más urbanizadas fue regular. Se recomienda la elaboración de un plan de manejo costero debido a que, de continuar el crecimiento urbano y la actividad turística, se acentuará la tendencia negativa de los servicios.
\end{abstract}

Palabras clave: Servicios de regulación, playas, erosión costera, General Alvarado, Buenos Aires.

\begin{abstract}
Urbanization of coastal cities has altered the ecosystem services provision in littoral areas around the world. Beach regulating services have been intensely modified particularly in touristic areas. The objective of this study was to evaluate the state of regulating and natural perturbations services of the coastal ecosystem in the cities of Miramar and Mar del Sur (Argentine). Five beaches with different geomorphological characteristics and anthropic intervention were studied. Anthropic and natural indicators were selected for the evaluation of the state of the services. Services provision was good in beaches with minor antropic intervention, and was regular in beaches with high antropic intervention. The elaboration of a costal management program is considered necessary, because if the urbanization and tourism growth continues, the negative tendency of the regulating services will increase.
\end{abstract}

Keywords: Regulating services, beaches, coastal erosion, General Alvarado, Buenos Aires.

Instituto de Investigaciones Marinas y Costeras (IIMYC), FCEYN, UNMDP-CONICET. Instituto de Geología de Costas y del Cuaternario, FCEYN, UNMDP-CIC. Mar del Plata, Argentina. amerlott@mdp.edu.ar

CONICET. Centro de Investigaciones Geográficas y Socioambientales, Facultad de Humanidades (CIGSA-UNMdP). Mar del Plata, Argentina. eleonorav@mdp.edu.ar

Instituto de Investigaciones Marinas y Costeras (IIMYC), FCEYN, UNMDP-CONICET. Instituto de Geología de Costas y del Cuaternario, FCEYN, UNMDP-CIC. Mar del Plata, Argentina. gbertola@mdp.edu.ar 
Las primeras referencias al concepto de servicio ecosistémico se remontan a los años 70 y luego de un impulso durante la década de 1990, el término se consolidó mediante la Evaluación de los Ecosistemas del Milenio a partir del año 2003 (MEA, 2005, de Groot et al., 2010, La Notte et al., 2017). Desde entonces, el enfoque de los servicios ecosistémicos ha adquirido especial relevancia, tanto en ámbitos académicos como políticos y de gestión. Ha sido considerado como un marco conceptual de gran utilidad para abordar y demostrar los vínculos existentes entre las sociedades y los ecosistemas y la dependencia del bienestar humano de esta relación (de Groot et al., 2010). Sin embargo, a pesar de la gran importancia de los beneficios obtenidos por las sociedades a partir de los ecosistemas, se evidencia una falta de marcos teóricos-metodológicos que vinculen la investigación de servicios ecosistémicos con la planificación de espacios urbanos (Vásquez, 2016) y la gestión de recursos.

Los servicios ecosistémicos se definen como aquellos beneficios directos (servicios intermedios) e indirectos (servicios finales) que los ecosistemas brindan a las sociedades y que contribuyen a su bienestar humano (MEA, 2005, Fisher y Turner, 2008, Haines-Young y Potschin, 2010). Se clasifican en tres grandes grupos: de abastecimiento, de regulación y culturales (MEA, 2005). Los servicios dependen del buen estado de los procesos y estructura de los ecosistemas que, a su vez, determinan sus funciones (Maynard et al., 2010). Las funciones se definen como la capacidad que tienen los ecosistemas de brindar servicios y, por lo tanto, sus características definen el flujo de servicios. A partir de estas conceptualizaciones, es posible abordar el estudio de los servicios ecosistémicos, siguiendo una serie de pasos que van desde las estructuras y los procesos ecológicos generados por los ecosistemas, los servicios y beneficios que ofrecen y su impacto en el bienestar humano (Haines-Young y Potschin, 2010). Este enfoque, denominado Cascada (Figura $N^{\circ} 1$ ), es de gran utilidad porque pone en evidencia que para obtener un flujo continuo de servicios ecosistémicos es necesario proteger y conservar los ecosistemas, con la estructura y procesos que le dan sustento (Maynard et al., 2010).

Figura $\mathrm{N}^{\circ} 1$

Enfoque cascada de los servicios ecosistémicos.

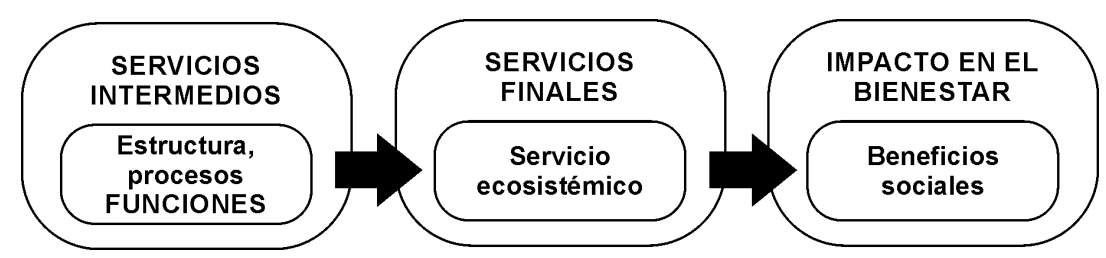

Fuente: Adaptado de Haines-Young y Potschin (2010).

La Evaluación de los Ecosistemas del Milenio clasificó los ecosistemas mundiales en seis grandes grupos (MEA, 2005). Entre ellos, los ecosistemas costeros constituyen uno de los sistemas más complejos y frágiles, pero también uno de los más dinámicos, interdependientes, productivos y diversos del planeta. Debido a ello ofrecen una numerosa cantidad de servicios ecosistémicos de los que las sociedades se benefician. Entre los servicios brindados por el ecosistema costero pueden mencionarse los de abastecimiento (pesca, agricultura), de regulación (climática, morfo-sedimentaria o control de la erosión, de perturbaciones naturales o protección contra tormentas, hídrica) y culturales (actividades recreativas y turismo, identidad cultural, disfrute esté- 
tico y paisajístico) (MEA, 2005, Barbier et al., 2011, Barragán y Chica, 2013, Christie et al., 2015). El $62 \%$ de los ecosistemas costeros del mundo se ha visto degradado y continúa esa tendencia debido a cambios en el uso del suelo, alteración de los ciclos biogeoquímicos, destrucción y fragmentación de hábitats, introducción de especies y alteración en las condiciones climáticas (cambios en las precipitaciones, patrones de tormentas, temperaturas extremas) (Brenner et al., 2010, de Groot et al., 2010, Barbier et al., 2011, Barragán y Borja, 2011, Barragán y Chica, 2013).

El ecosistema costero se compone de una serie de tipos operativos que permiten realizar un análisis pormenorizado y completo del mismo. Entre ellos pueden mencionarse playas, médanos, marismas, humedales y aguas costeras (MEA, 2005, Barbier et al., 2011, Epanchin-Niell et al., 2017). Las playas se definen como formaciones sedimentarias sujetas a una dinámica muy reactiva que se encuentran condicionadas por la presencia humana e infraestructuras turísticas (Barragán y Chica, 2013). Son particularmente importantes para la protección contra tormentas, control de la erosión, turismo y recreación, captación y purificación del agua, mantenimiento de la biodiversidad y provisión de materias primas, entre otras (Marshall et al., 2014, Alexandrakis et al., 2015, Harris et al., 2015). Las principales presiones o impulsores de cambio sobre el ecosistema playa se derivan de impactos del cambio climático (aumento del nivel del mar, incremento de precipitaciones y evaporación, intensidad y frecuencia de tormentas), del incremento de construcciones como defensas o infraestructura turística y de la contaminación por desechos (residuos sólidos, aguas residuales), entre otros (Marshall et al., 2014).

Los estudios sobre los servicios ecosistémicos brindados por las playas son escasos. La mayor parte de ellos han evaluado los servicios de abastecimiento y culturales provistos por los ecosistemas costeros (Spalding et al., 2014, Christie et al., 2015) o se han centrado en el análisis y valoración por parte de los usuarios de las playas, de los servicios de recreación y turismo (Alexandrakis et al., 2015, Harris et al., 2015). En cuanto a los servicios de regulación, pueden mencionarse los trabajos que realizaron evaluaciones sobre el impacto de tormentas, tsunamis, huracanes y eventos extremos en los ecosistemas costeros, tanto en el aspecto físico como en la valoración económica de las consecuencias de dichos eventos sobre las sociedades afectadas (Brander et al., 2006, Turner et al., 2007, Barbier et al., 2011, Epachin-Niell et al., 2017). Los estudios sobre los servicios de regulación específicamente sobre el ecosistema playa son más escasos. Los trabajos que predominan son los que realizan valoraciones económicas y sociales sobre la importancia de las playas en el mantenimiento de procesos ecológicos y las consecuencias de su deterioro (Schlacher et al., 2008), sobre las pérdidas monetarias ocasionadas por el impacto de eventos extremos como huracanes o tsunamis (Meixler, 2017), así como el costo monetario que ocasiona la pérdida del servicio de protección contra tormentas e inundaciones (Liquete et al., 2013, Barbier, 2015). En menor medida se han desarrollado metodologías para seleccionar indicadores ecosistémicos y humanos relevantes para evaluar la condición del ecosistema playa (Marshall et al., 2014).

Si bien todos los servicios proporcionados por el ecosistema playa son importantes, los servicios de regulación morfo-sedimentaria y de perturbaciones naturales (también denominados control de la erosión y protección contra tormentas) son de los más valiosos, en especial ante tormentas extremas, tsunamis y aumento del nivel del mar (Marshall et al., 2014, Spalding et al., 2014). En la provisión de estos servicios tiene un papel indispensable el estado del ecosistema, el cual depende, a su vez, de su funcionamiento general y de su capacidad de absorber las diferentes formas de concentración de energía procedentes de medio marino, así como de la redis- 
tribución sedimentaria de la cual depende su salud e integridad (Barbier, 2012; Barragán y Chica, 2013). Es decir, que del buen funcionamiento de la estructura y procesos (funciones) del ecosistema, depende el flujo de servicios y con ello, los beneficios que pueden percibir las sociedades (Figura $\mathrm{N}^{\circ} 2$ ).

Figura $\mathrm{N}^{\circ} 2$

Funciones y beneficios generados por los servicios de regulación del ecosistema playa.

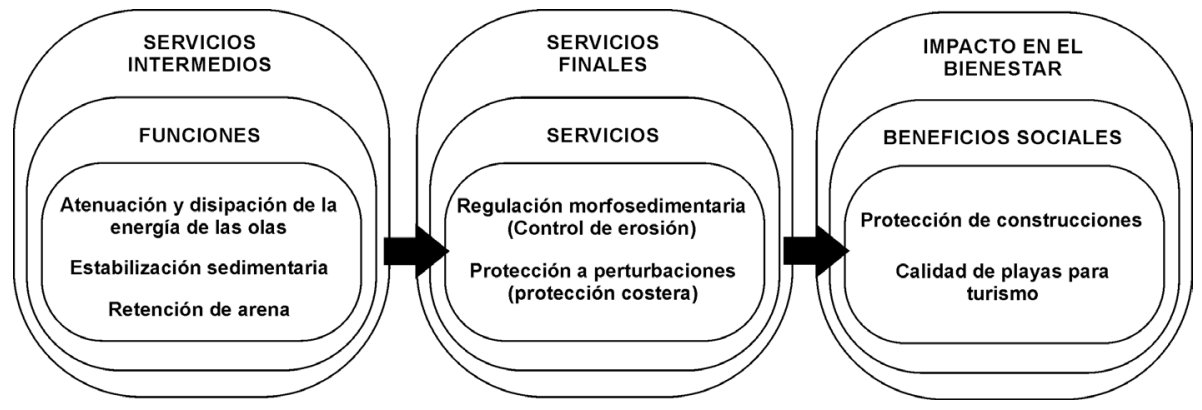

Fuente: Adaptado de Haines-Young y Potschin (2010).

La regulación morfodinámica de las playas depende, principalmente, de las condiciones del oleaje y del intercambio de sedimentos entre el médano, la playa distal y la barra litoral. No obstante, el estado de la playa se ve fuertemente influenciado por las actividades humanas, especialmente en aquellas áreas en las cuales se desarrolla la práctica turística. En la provincia de Buenos Aires (Argentina), los ecosistemas costeros se encuentran entre los sistemas más amenazados debido a las presiones y alteraciones que han sufrido a lo largo de la historia (Dadón, 2011, Merlotto y Bértola, 2008 y 2009, Verón y Barragán, 2015). El intenso proceso de urbanización de las ciudades costeras, junto con la sobreexplotación del capital natural han alterado y degradado estos ecosistemas. Las tormentas, acompañadas generalmente de ondas de tormenta positivas y fuertes vientos, han sido identificadas como uno de los principales factores naturales que afectan el flujo de servicios de regulación morfo-sedimentaria de las playas ocasionado procesos erosivos de diversa magnitud (Schnack et al., 1998, Fiore et al., 2009, Merlotto y Bértola, 2009, Merlotto et al., 2013). Además, han afectado la provisión del servicio de regulación a perturbaciones naturales, ocasionando el deterioro y destrucción de infraestructura balnearia y residencial en numerosas ciudades de la provincia de Buenos Aires (Pousa et al., 2007, Merlotto y Bértola, 2009, Fiore et al., 2009, Codignotto et al., 2012, Merlotto et al., 2013).

En Argentina se han desarrollado escasos análisis sobre los servicios provistos por los ecosistemas costeros y no se han realizado trabajos sobre playas y los servicios de regulación (Verón y Barragán, 2015). Es por ello que el objetivo del presente trabajo fue evaluar el estado y tendencia de los servicios de regulación morfo-sedimentaria y a perturbaciones naturales del ecosistema playa en un sector de la provincia de Buenos Aires. El área de estudio comprende distintas playas de las ciudades de Miramar y Mar del Sur, partido de General Alvarado (Figura No 3). Ambas constituyen localidades turísticas con una importante atracción de visitantes provenientes de toda la provincia. Si bien no existen cifras oficiales sobre la cantidad de turistas que arriban a las localidades, las estimaciones realizadas arrojan que a Miramar llegan alrededor de 160000 turistas por temporada estival, mientras que a Mar del Sur 5000 (Comunicación personal, Secretaría de Turis- 
mo). A pesar de localizarse en un partido dedicado principalmente a la actividad agropecuaria, el turismo de sol y playa desarrollado en el área de estudio es la principal fuente de ingresos de las localidades. Por lo tanto, el estado de las playas constituye una condición determinante para el desarrollo de dicha actividad, principal motor económico del área.

Figura $\mathrm{N}^{\circ} 3$

Ubicación del área de estudio

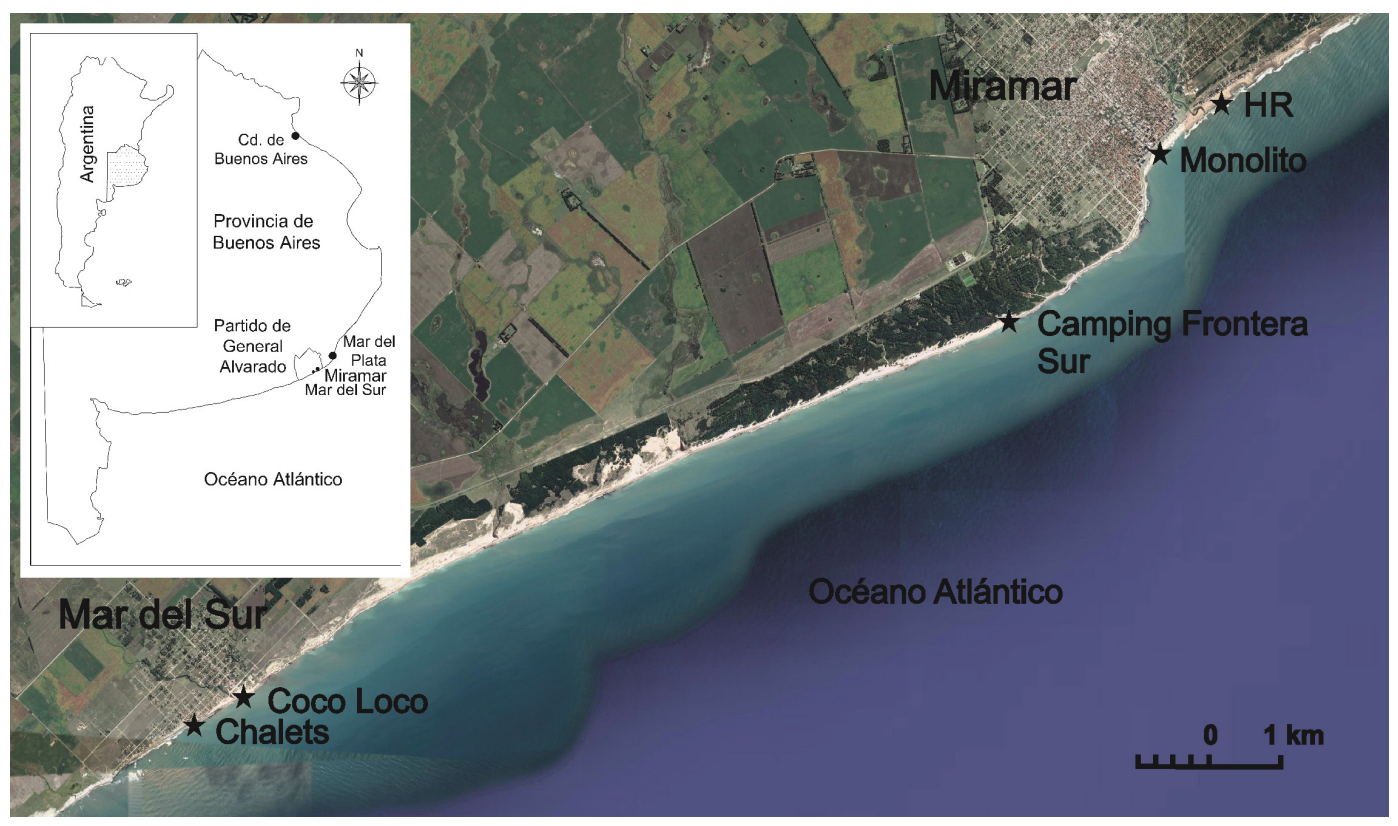

Fuente: elaboración personal.

\section{Área de estudio}

Miramar y Mar del Sur son localidades turísticas de la provincia de Buenos Aires (Figura № 3), con una población estable de 19553 y 298 habitantes, respectivamente (INDEC, 2010). En ellas se desarrolla el turismo de sol y playa, el cual se centra en la valorización del ecosistema y de sus componentes y procesos. Dada la estacionalidad que caracteriza dicha actividad, el área es altamente sensible a los cambios políticos, económicos y ambientales (Varisco, 2004).

A partir de sus fundaciones y a lo largo de su historia, ambas localidades se desarrollaron como centros turísticos. Desde los años 50 del siglo XX se fue afianzando la actividad, impulsada por obras e infraestructuras generadas con tal fin (Brugueras, 2004) y políticas nacionales orientadas al turismo masivo. De esta forma, al igual que en la mayor parte de las ciudades costeras de la provincia, la apropiación del ecosistema costero por urbanizaciones turísticas ha sido el principal proceso desarrollado en el área de estudio (Dadón, 2011). En especial, este proceso se caracterizó por la ocupación intensiva del frente costero ante la valorización social del ecosistema litoral, trayendo como consecuencia el deterioro ambiental. 
Para el presente trabajo, se seleccionaron cinco playas a lo largo del área, caracterizadas por su diferente geomorfología y por estar sujetas a diversos grados de urbanización e intervención antrópica. De Sur a Norte, las playas son Chalets y Coco Loco en la localidad de Mar del Sur y Frontera Sur, Monolito y HR en Miramar (Figura $\mathrm{N}^{\circ} 3$ ).

La costa presenta una orientación SW-NE, en coincidencia con la dirección predominante de la deriva litoral (Perillo et al., 2005). La marea es mixta, predominantemente semidiurna y la amplitud media es para la ciudad vecina de Mar del Plata de 0,98 m (SHN, 2016). Los vientos predominantes en la región provienen de los sectores $\mathrm{N}$ y NW y la velocidad media del viento es de 17,8 km h-1 (Merlotto y Piccolo, 2009). Las mayores velocidades corresponden en el sur del área de estudio a los vientos del SW y S, rotando hacia el SE al norte de la misma. En cuanto a la evolución de la línea de costa, Marcomini y López (1999) estimaron un proceso erosivo con un ritmo de retroceso de 0 a 1,54 m/año para el período 1956-1997 en ciertos sectores del partido. La extracción minera de arena fue considerada como una de las causas principales. Isla et al. (1997) estudiaron la morfodinámica de las playas de Mar del Sur, aunque no se han realizado estudios posteriores. Un relevamiento de la playa infralitoral efectuado en el año 2003 con el objetivo de estudiar la disponibilidad de sedimentos para una posible alimentación artificial de estas costas (Isla, 2003), estimó que la playa sumergida frente al área de estudio presenta acumulaciones de arena compuestas por arena muy fina y son muy escasas. Asimismo, las plataformas de abrasión sobre las cuales se desarrollan las playas del sur del área de estudio, se continúan hacia el fondo marino como un relieve irregular con escalones que superan los 1,5 m.

\section{Metodología}

El presente estudio se basó en la propuesta teórico-metodológica de la Evaluación de los Ecosistemas del Milenio (MEA, 2005), ampliamente utilizada en el mundo para evaluar los servicios que brindan los ecosistemas y su relación con el bienestar humano. Dicha metodología permite identificar los ecosistemas operativos y efectuar la clasificación de servicios en forma práctica, con el fin de evaluarlos y realizar propuestas de gestión. Además, se incorporó al estudio el enfoque de cascada de Haines-Young y Potschin (2010). Éste es empleado por la Agencia Europea de Medio Ambiente, entre otros, como marco de referencia para llevar a cabo la Clasificación Internacional Común para los Servicios de los Ecosistemas (CICES) (La Notte et al., 2017). A partir del enfoque cascada, se evaluaron las funciones ecosistémicas del área de estudio y, a partir de ello, se determinó el flujo de servicios. De esta forma, se complementó el análisis de los servicios ecosistémicos desde las estructuras y procesos ecológicos hasta el bienestar humano.

Para abordar la evaluación del estado de los servicios de regulación morfo-sedimentaria y a perturbaciones naturales del ecosistema operativo playa se seleccionaron indicadores que permitieron evaluar las funciones ecosistémicas y, de esta forma, determinar el flujo de servicios. Las funciones identificadas fueron atenuación y disipación de la energía de las olas, estabilización sedimentaria y retención de arena. Los indicadores empleados fueron grado de intervención antrópica, presencia de defensas costeras, geomorfología costera, ancho de playa, pendiente de playa frontal y balance sedimentario. Su selección y estudio se basó en trabajos de campo y las categorías de cada indicador fueron establecidas de acuerdo con las características del área de estudio. 
Cada indicador fue dividido en 5 categorías. Las mismas fueron establecidas cuantitativamente y cuando no fue posible, se realizó una descripción y clasificación cualitativa (Cuadro $N^{\circ} 1$ ): se asignó un valor del 1 al 5 a cada categoría, en el cual 1 representa muy buena condición del indicador y 5 , muy mala. Luego, los indicadores fueron combinados para obtener el estado del servicio:

$$
\text { Estado }=\left(i_{1}+i_{2}+i_{3}+\ldots+i_{n}\right) / n
$$

En la ecuación, i corresponde al indicador y $\mathrm{n}$ al número de indicadores utilizados. Se determinaron intervalos equivalentes para obtener el valor del estado del servicio desde 1 a 5 , correspondiendo 1: muy buen estado, 2: buen estado, 3: estado moderado, 4: estado regular y 5: estado malo del servicio del ecosistema. Los indicadores empleados (Cuadro $N^{\circ} 1$ ) son definidos a continuación.

- Grado de intervención antrópica. Se establecieron 5 categorías de intervención antrópica de acuerdo con el porcentaje de ocupación del frente costero y la cobertura vegetal (considerando $100 \mathrm{~m}$ a ambos lados de cada perfil) (Cuadro $\mathrm{N}^{\circ}$ 1): sin intervención, escasa, moderada, alta y frente totalmente urbanizado. Se trabajó con imágenes satelitales Quickbird del año 2015 y 2016 (Google Earth ${ }^{\circledR}$ ) y se complementó con fotografías y observaciones visuales durante los relevamientos de campo.

Cuadro $\mathrm{N}^{\circ} 1$

Indicadores y categorías del servicio de regulación morfo-sedimentaria y a perturbaciones naturales.

\begin{tabular}{|c|c|c|c|c|c|}
\hline \multirow[b]{2}{*}{ Indicador } & \multicolumn{5}{|c|}{ Categorías } \\
\hline & $\begin{array}{c}\text { Muy buena } \\
\text { (1) }\end{array}$ & Buena (2) & Moderada (3) & Mala (4) & Muy mala (5) \\
\hline $\begin{array}{l}\text { Grado de } \\
\text { intervención } \\
\text { antrópica }\end{array}$ & $\begin{array}{c}\text { Frente } \\
\text { costero sin } \\
\text { intervención }\end{array}$ & $\begin{array}{l}\text { Frente } \\
\text { costero con } \\
\text { escasa inter- } \\
\text { vención }\end{array}$ & $\begin{array}{c}\text { Frente } \\
\text { costero con } \\
\text { moderada } \\
\text { intervención }\end{array}$ & $\begin{array}{l}\text { Frente costero } \\
\text { con alta inter- } \\
\text { vención }\end{array}$ & $\begin{array}{l}\text { Frente coste- } \\
\text { ro totalmente } \\
\text { urbanizado }\end{array}$ \\
\hline $\begin{array}{l}\text { Presencia } \\
\text { de defensas } \\
\text { costeras }\end{array}$ & Sin defensas & $\begin{array}{l}\text { Una defensa } \\
\text { a más de } 150 \\
\text { m }\end{array}$ & $\begin{array}{c}\text { Una defensa } \\
\text { a menos de } \\
150 \mathrm{~m}\end{array}$ & $\begin{array}{c}\text { Entre defensas } \\
\text { a más de } 150 \\
\text { m }\end{array}$ & $\begin{array}{c}\text { Entre defen- } \\
\text { sas a menos } \\
\text { de } 150 \mathrm{~m}\end{array}$ \\
\hline $\begin{array}{l}\text { Geomorfología } \\
\text { costera }\end{array}$ & $\begin{array}{l}\text { Playas de } \\
\text { arena con } \\
\text { médanos } \\
\text { vivos o semi- } \\
\text { fijos }\end{array}$ & $\begin{array}{l}\text { Playas de } \\
\text { arena con } \\
\text { acantila- } \\
\text { dos bajos y } \\
\text { cubiertos por } \\
\text { médanos }\end{array}$ & $\begin{array}{c}\text { Playas de } \\
\text { arena con } \\
\text { acantila- } \\
\text { dos altos y } \\
\text { desarrollo de } \\
\text { playa }\end{array}$ & $\begin{array}{l}\text { Acantilados } \\
\text { activos con } \\
\text { plataformas } \\
\text { de abrasión y } \\
\text { escaso desa- } \\
\text { rrollo de playa }\end{array}$ & $\begin{array}{c}\text { Playa are- } \\
\text { nosa sin } \\
\text { espaldón } \\
\text { (urbanizada) }\end{array}$ \\
\hline $\begin{array}{l}\text { Ancho de playa } \\
(\mathrm{m})\end{array}$ & $\geq 146$ & 121 a 145 & 96 a 120 & 71 a 95 & $\leq 70$ \\
\hline $\begin{array}{l}\text { Pendiente de la } \\
\text { playa frontal (\%) }\end{array}$ & $\leq 3$ & 3,1 a 5 & 5,1 a 7 & 7,1 a 9 & $\geq 9,1$ \\
\hline $\begin{array}{l}\text { Balance } \\
\text { sedimentario }\end{array}$ & Muy positivo & Positivo & Estable & Negativo & Muy negativo \\
\hline
\end{tabular}

Fuente: elaboración personal. 
- Presencia de defensas costeras. Las 5 categorías fueron establecidas sobre la base de la influencia de las defensas en las playas, de acuerdo con su proximidad y por lo tanto en la funcionalidad proporcionada en cada playa seleccionada (Cuadro $\mathrm{N}^{\circ} 1$ ). El menor valor correspondió para las playas sin defensas, 2 para las playas con una defensa a más de $150 \mathrm{~m}, 3$ para las playas con un defensa a menos de $150 \mathrm{~m}$, 4 para las playas entre defensas a más de $150 \mathrm{~m}$ entre ambas y el valor más alto correspondió a las playas con defensas muy próximas entre sí (menos de $150 \mathrm{~m}$ ).

- Geomorfología costera. Las geoformas predominantes en un sector costero y sus características, permiten inferir la evolución y cambios en el paisaje y su posible degradación a partir de los procesos erosivos y diversas condiciones energéticas. Para establecer las categorías del indicador, se consideró que la menor vulnerabilidad a la degradación costera la poseen las playas arenosas provistas de médanos y la mayor, las playas totalmente urbanizadas, sin rasgos geomorfológicos en su espaldón (Cuadro № 1). Para evaluar el indicador, además de los trabajos de campo se emplearon cartas topográficas (E: 1:50000, Instituto Geográfico Nacional) e imágenes satelitales Quickbird del año 2015 y 2016 (Google Earth ${ }^{\circledast}$ ).

- Ancho de playa. El ancho, junto a otras características de la playa, influye en la frecuencia en que la marea y las olas cubren la playa y alcanzan su parte posterior, pudiendo ocasionar diferentes grados de erosión durante las tormentas. Para determinar las categorías, se consideró desde una playa angosta menor a los $70 \mathrm{~m}$ y cada categoría se incrementa de a $25 \mathrm{~m}$, ya que las playas que sobrepasan los $120 \mathrm{~m}$ de ancho son escasas en el área de estudio y en toda la provincia de Buenos Aires (Cuadro $N^{\circ} 1$ ).

- Pendiente de la playa frontal. La pendiente de la playa frontal es la relación existente entre el desnivel de la cresta de la berma de playa (máxima altura a la que llegan las pleamares) y las bajamares y la distancia horizontal entre ambos puntos. Puede expresarse en porcentaje o en grados y se clasifica de acuerdo con su intensidad, desde pendientes suaves a muy violentas (IGM, 1976). De acuerdo a las pendientes observadas se determinaron las categorías (Cuadro $N^{\circ} 1$ ).

- Balance sedimentario. El balance sedimentario a lo largo de un período permite apreciar las condiciones sedimentarias bajo la cual se encuentra la playa: erosión, estabilidad o acreción. Del 1 al 5, las categorías fueron establecidas desde un balance sedimentario muy positivo a uno muy negativo (Cuadro $N^{\circ} 1$ ).

Los indicadores geomorfológicos fueron evaluados a partir de 16 trabajos de campo, efectuados con una periodicidad estacional: 25/03/13, 25/06/13, 04/10/13, 16/12/13, 27/03/14, 30/06/14, 10/10/14, 16/04/15, 29/06/15, 26/10/15, 22/12/15, 04/04/16, 16/06/16, 16/09/16, 12/12/16 у 23/03/17. Durante los mismos se realizaron perfiles de playa perpendiculares a la línea de costa utilizando un teodolito NIKON NT2A, desde un punto fijo hasta una cota de $0,5 \mathrm{~m}$ por debajo del nivel de bajamar.

En laboratorio se procesaron los datos de campo mediante técnicas estadísticas estándar para reconstruir los perfiles de playa y determinar el ancho de playa, la pendiente de playa frontal y el balance sedimentario. El ancho de playa se obtuvo midiendo la distancia reducida al plano desde el pie de médano (o pie de acantilado) hasta la bajamar durante las mareas de sicigia. La pendiente de la playa frontal se determinó mediante la siguiente ecuación:

$$
P=C_{1}-C f / L .100
$$

donde $\mathrm{C}_{1}$ es la cota de la cresta de la berma, $\mathrm{Cf}$ es la cota del último punto del perfil y $\mathrm{L}$ es la distancia entre ambos puntos. 
El balance de sedimentos se calculó mediante una técnica convencional (Fox y Davis, 1978) ampliamente utilizada en el estudio de las playas bonaerenses (Bértola, 2001, Merlotto et al., 2014). El volumen de arena se calculó como el área bajo el perfil, desde el primer punto del perfil y hasta el nivel base definido por la última cota del mismo, considerando una continuidad lateral de $5 \mathrm{~m}$ a ambos lados del perfil.

Para analizar la respuesta del ecosistema y de sus servicios a las alteraciones externas, se consideró como un impulsor de cambio importante en el área, el efecto sobre las playas de los temporales de oleaje. Para ello se seleccionaron los relevamientos topográficos del 16 de abril y del 29 de junio de 2015 debido a que se observaron cambios morfológicos que se destacaban con respecto a los perfiles medios de las playas. Para el estudio del oleaje del período elegido se procesaron los datos de ola del modelo WAVEWATCH III (SMN). Se consideró como oleaje asociado a temporal, una altura de ola significativa media diaria $\geq 1,5 \mathrm{~m}$ (Merlotto et al., 2010 y 2013).

\section{Resultados y discusión}

Los servicios de regulación morfo-sedimentaria y a perturbaciones naturales presentaron para el conjunto del área estudiada, un estado general de regular a bueno (Cuadro $N^{\circ} 2$ ). En las playas de Mar del Sur, Chalets y Coco Loco, el estado de los servicios es moderado y bueno, respectivamente. Ambas presentan un frente costero con moderada intervención. La playa Chalets posee un ancho medio de $68 \mathrm{~m}$ y se desarrolla al pie de acantilados activos de baja altura (Figura $\mathrm{N}^{\circ}$ $4 a$ ), que aumentan hasta los $3 \mathrm{~m}$ hacia el Norte (Figura $\mathrm{N}^{\circ} 4 \mathrm{~b}$ ), sobre los cuales se han construido viviendas con vista al mar. La playa arenosa se desarrolla sobre una extensa plataforma de abrasión conformada por limos entoscados del Plioceno y Pleistoceno (Isla, 2003). Ésta se presenta frecuentemente desprovista de sedimentos, los cuales se encuentran en continua disminución, por lo tanto, le corresponde los mayores valores en estos indicadores. No posee defensas costeras. Por todo ello, a Chalets le corresponden valores dispares en los indicadores (Cuadro $N^{\circ} 2$ ), que permitieron inferir que sus funciones (procesos y estructura) proveen un flujo moderado de servicios ecosistémicos.

La playa Coco Loco (Figuras $N^{\circ} 4 c$ y d) con un ancho medio de $155 \mathrm{~m}$, es la más extensa de las estudiadas. Se desarrolla contigua a un pequeño campo de médanos semifijos y hacia el Norte se emplaza un pequeño espigón. Presenta un balneario que provee servicios gastronómicos y de ocio a los veraneantes. Su morfología y balances sedimentarios se ven afectados por la desembocadura del Arroyo La Tigra, cuyo caudal y morfología depende de las precipitaciones (Figuras $\mathrm{N}^{\circ} 4 \mathrm{c}$ y d). A pesar de las importantes variaciones sedimentológicas observadas entre los relevamientos, el balance sedimentario es estable. Los valores de los indicadores en esta playa han resultado bajos en su mayor parte (Cuadro $N^{\circ} 2$ ), por lo tanto, el estado de los servicios es bueno.

Aproximadamente $15 \mathrm{~km}$ hacia el Norte, la playa Frontera Sur se desarrolla al pie de un campo de médanos semi-forestados pertenecientes al Vivero Florentino Ameghino (Figura $\mathrm{N}^{\circ} 4 \mathrm{e}$ ). De las playas estudiadas, es la que posee el menor grado de intervención antrópica. No posee defensas costeras ni infraestructura balnearia, correspondiéndole los valores más bajos en estos indicadores. Posee un ancho medio de $89 \mathrm{~m}$ y se encuentra en una condición sedimentaria estable. Es por ello que la playa Frontera Sur posee un estado bueno de los servicios ecosistémicos evaluados. 


\section{Cuadro $\mathrm{N}^{\circ} 2$}

Valores de los indicadores y estado de los servicios de regulación en las playas estudiadas.

\begin{tabular}{|l|c|c|c|c|c|}
\hline \multirow{2}{*}{ Indicador } & \multicolumn{5}{|c|}{ Playas } \\
\cline { 2 - 6 } & Chalets & $\begin{array}{c}\text { Coco } \\
\text { Loco }\end{array}$ & Frontera Sur & Monolito & HR \\
\hline Grado de intervención antrópica & 3 & 3 & 2 & 5 & 5 \\
\hline Presencia de defensas costeras & 1 & 2 & 1 & 5 & 4 \\
\hline Geomorfología costera & 4 & 2 & 1 & 5 & 5 \\
\hline Ancho de playa (m) & 5 & 1 & 4 & 3 & 3 \\
\hline Pendiente de la playa frontal (\%) & 2 & 3 & 3 & 4 & 4 \\
\hline Balance sedimentario & 5 & 3 & 3 & 2 & 2 \\
\hline $\begin{array}{l}\text { Estado de los servicios de regu- } \\
\text { lación }\end{array}$ & moderado & bueno & bueno & regular & regular \\
\hline
\end{tabular}

Fuente: elaboración personal.

Hacia el Norte, en el centro urbanizado de Miramar, las playas Monolito y HR poseen infraestructuras balnearias de mampostería de envergadura y defensas costeras, por lo tanto, poseen los mayores valores en estos indicadores. Monolito (Figura $\mathrm{N}^{\circ} 4 \mathrm{f}$ ) tiene un ancho medio de $109 \mathrm{~m}$ y se localiza entre pequeños espigones constituidos por rocas. La playa HR (Figura $\mathrm{N}^{\circ} \mathrm{4g}$ ) posee una extensión promedio de $114 \mathrm{~m}$ y también se localiza entre escolleras conformadas por rocas, pero en forma de T y más distanciadas. Durante la primavera y verano estas playas son preparadas para el turismo mediante el alisado de la playa distal con maquinarias y posterior instalación de carpas. Como los balances sedimentarios resultaron positivos, les corresponden a ambas playas los menores valores del área de estudio en esta categoría. No obstante, el estado de los servicios ecosistémicos evaluados en estas playas es regular, debido al intenso grado de urbanización que ha modificado las características geomorfológicas de las mismas y su capacidad de amortiguar las perturbaciones naturales.

Los cambios sufridos por las playas debido a los temporales de oleaje fueron evaluados al ser considerados como uno de los impulsores de cambio de los servicios ecosistémicos brindados por las playas. En el período seleccionado, otoño de 2015 (16 de abril al 29 de junio), se observaron cambios morfológicos que se destacan con respecto a los perfiles medios de las playas, los cuales fueron ocasionados por frecuentes temporales de oleaje. El $40 \%$ de los días, el oleaje presentó una altura de ola significativa media diaria superior a 1,5 m, de los cuales el 7\% superó los $3 \mathrm{~m}$ (Figura $\mathrm{N}^{\circ} 5$ ). La mitad de los días con oleaje de temporal se manifestó durante el mes de junio. Entre los principales cambios observados en la morfología de las playas (Figura $N^{\circ} 6$ ), se destaca la migración de las barras y formación de escarpas (Chalets, Figura $N^{\circ} 6$ ), erosión de la berma y reducción de las pendientes de playa (Coco loco, Frontera Sur y HR, Figura $N^{\circ} 6$ ). En la playa Monolito (Figura $\mathrm{N}^{\circ} 6$ ) los cambios morfológicos fueron menores. Los balances sedimentarios fueron negativos en todas las playas menos en Chalets, que se mantuvo estable. En el período siguiente, las playas continuaron perdiendo sedimentos, aunque el balneario HR, situado deriva 
arriba, se recuperó. La playa Coco Loco también se recuperó, pero debido a la reducción de las dimensiones del arroyo que la atraviesa.

Figura $\mathrm{N}^{\circ} 4$

Playas estudiadas. a) Playa Chalets vista desde el Norte y b) desde el Sur, c) Playa Coco Loco vista desde la costa, con presencia o d) ausencia del Arroyo La Tigra. e) Playa Frontera Sur vista desde el Sur, f) Playa Monolito vista desde el Norte y g) Playa HR, vista desde el Sur.
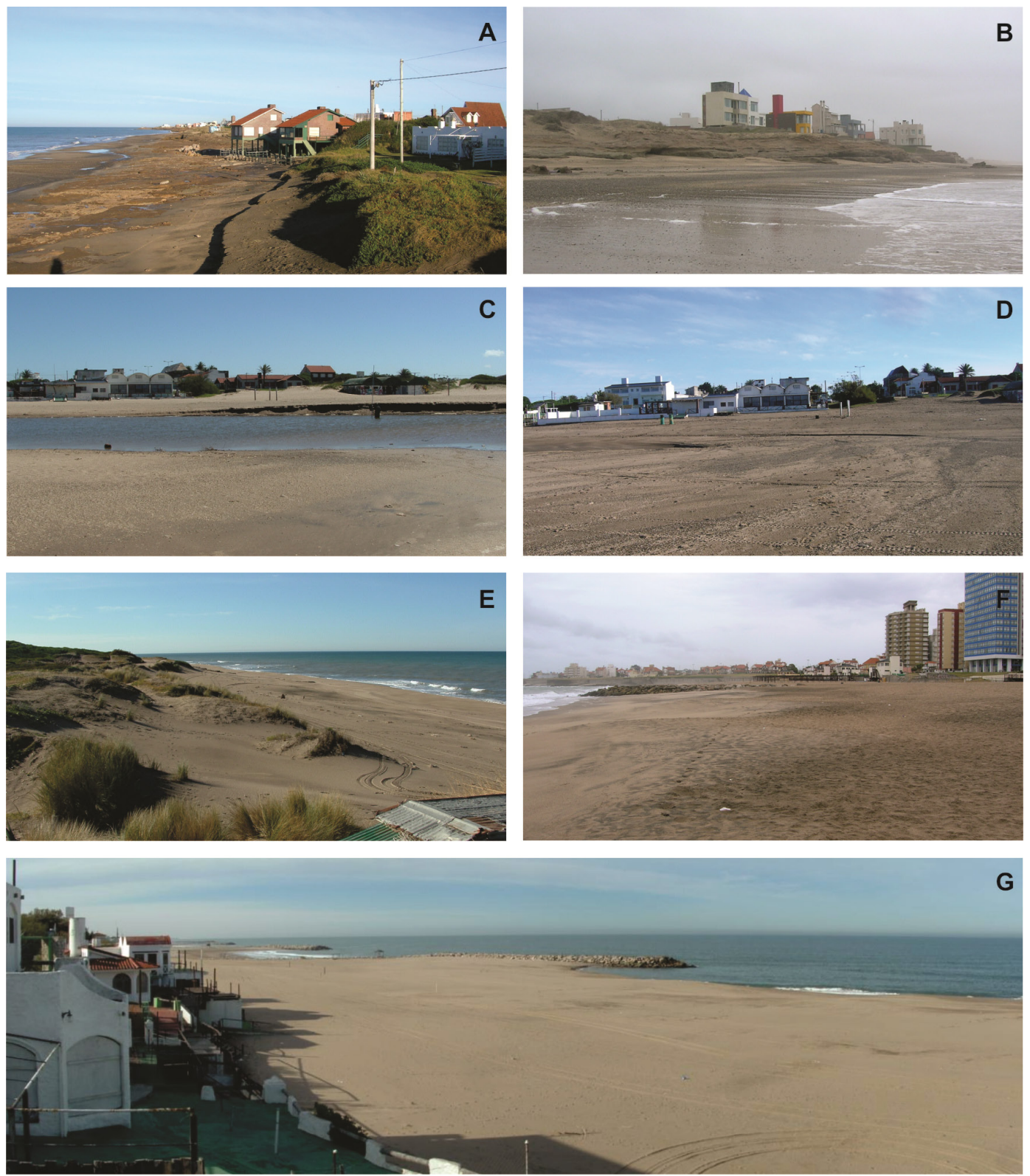

Fuente: archivo personal de los autores. 
Figura $N^{\circ} 5$

Altura significativa media diaria de ola (16/04/2015 al 29/06/2015).

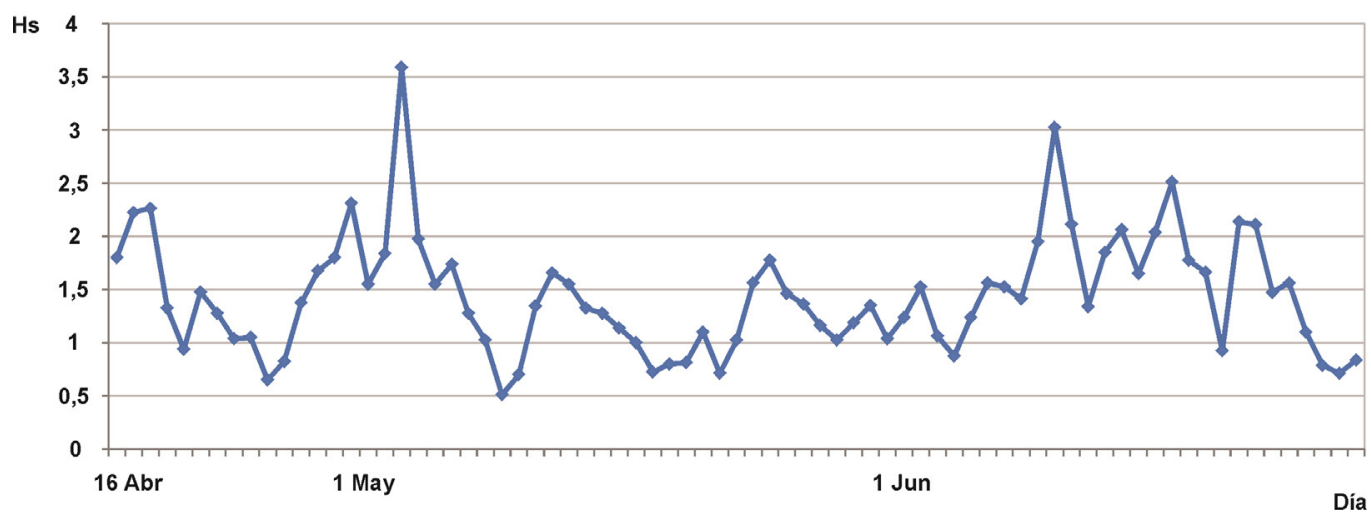

Fuente: Servicio Meteorológico Nacional (SMN).

Las playas arenosas presentan una gran variabilidad espacial y temporal a lo largo de la costa influenciadas por gran cantidad de variables (ciclos estacionales, tormentas, entre otros) (Merlotto et al., 2013). La morfología de playa depende principalmente de las condiciones del oleaje y del intercambio de sedimentos entre el médano, la playa distal y la barra litoral. Los cambios morfológicos y volumétricos de las playas pueden estar más relacionados con la frecuencia e intensidad de las tormentas en lugar de seguir un comportamiento estacional cíclico (Anfuso et al., 2006).

El ecosistema playa, al mantener su estructura y procesos naturales, permite que las funciones de atenuación y disipación de olas, estabilización sedimentaria y retención de arena se desarroIlen ofreciendo un servicio de absorción de parte de la energía marina y con ello, los servicios de regulación morfo-sedimentaria y a perturbaciones naturales. Esto se evidencia, en especial, ante perturbaciones externas como los eventos de temporales (Burkhard et al., 2012, Harris et al., 2015). Las playas que mantienen su funcionamiento natural proveen estos servicios ecosistémicos ya que controlan los procesos erosivos mediante la redistribución de los sedimentos y la amortiguación de las perturbaciones o impulsoras de cambio registradas en el frente costero, manteniendo la integridad del ecosistema (Barragán y Chica, 2013). Una playa con alta intervención antrópica puede sufrir el descenso de su capacidad de provisión de servicios debido a la alteración de su dinámica natural. Cuando la dinámica de las playas se ve afectada, no se amortiguan las perturbaciones registrándose la pérdida de superficie, pérdida del volumen de arena y cambios en el perfil de playa, así como daños en infraestructuras e instalaciones localizadas sobre el borde costero (Viles y Spencer, 1995).

Según Ferreira (2006) una playa puede ser afectada de diversas maneras bajo el impacto de uno o de varios eventos de tormenta sucesivos y la erosión es mayor cuando la frecuencia de las tormentas excede el tiempo de recuperación de la playa para una tormenta individual (Morton et al., 1995). La ocurrencia de sucesivos temporales puede dificultar la recuperación de la playa ya que los sedimentos localizados en la playa infralitoral luego de un temporal, pueden ser retirados de la misma y ser transportados por deriva litoral en lugar de retornar a la playa en las condiciones de oleaje calmo, favoreciendo el proceso erosivo. Asimismo, los cambios morfológicos 
observados en cada perfil de playa luego de continuos temporales de oleaje, están vinculados con la situación previa a los mismos y al grado de intervención antrópica en cada perfil. Estos factores afectan la capacidad de respuesta y recuperación de las playas (Merlotto et al., 2013). Los resultados hallados en las playas estudiadas evidencian que el área de estudio registró luego del otoño de 2015 importantes pérdidas de arena en todas las playas (excepto en Chalets). Se ha observado que esta playa relativamente angosta y de fuerte comportamiento reflectivo, con un balance sedimentario aparentemente erosivo en los sectores supralitorales (erosión en la berma o playa distal), ha presentado acumulación en los sectores sublitorales (Figura $N^{\circ} 6$ ), resultando, por lo tanto, en un balance estable. Asimismo, la plataforma de abrasión expuesta en los últimos períodos, puede contribuir a reducir la energía del oleaje. Ejemplos similares se han observado en playas de bolsillo, también reflectivas, localizadas en los partidos de Mar Chiquita (Bértola et al., 2013) y Necochea (Merlotto et al., 2013). En los períodos siguientes, la recuperación de las playas fue lenta, aunque fue algo más rápida en las playas hacia el Norte (HR), provistas de defensas costeras las cuales estarían reteniendo la arena en tránsito sustraída en playas del Sur.

Figura $\mathrm{N}^{\circ} 6$

Perfiles de playa de los relevamientos del 16/04/2015 (pre temporales) y del 29/06/2015 (post temporales).

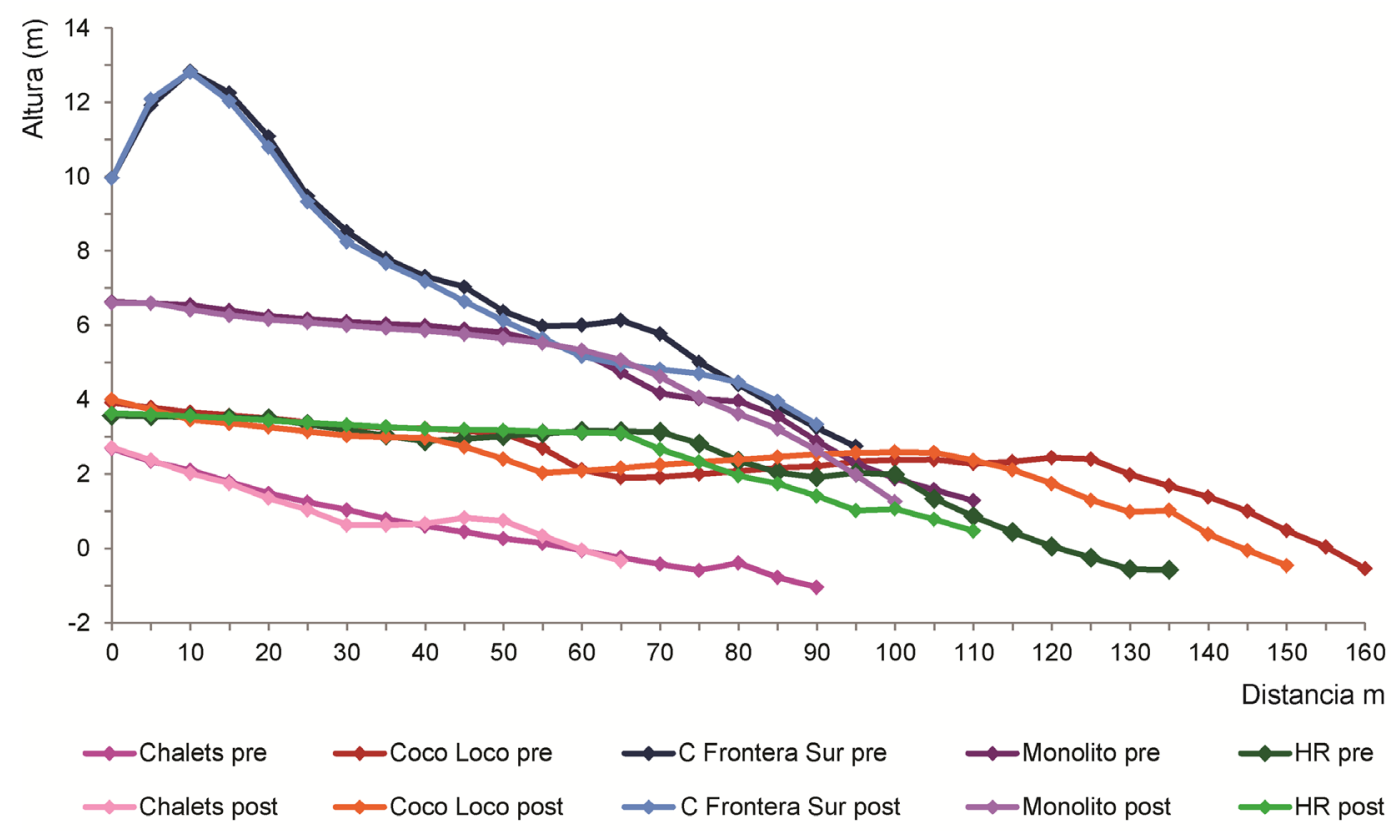

Fuente: elaboración personal.

Cuando los ecosistemas costeros se destruyen o degradan, pierden la capacidad de amortiguar de forma natural las perturbaciones y controlar la erosión. Como consecuencia, pueden producirse daños en las playas y en las infraestructuras, instalaciones y bienes situados en el borde costero. Es en ese momento cuando suelen realizarse obras de defensa con el fin de colaborar con la generación del servicio. Ello es posible mediante la intervención en los procesos, estructuras y las funciones ecosistémicas, como, por ejemplo, en la retención de sedimentos. Por 
tanto, puede encontrarse una playa con un buen estado del servicio de regulación gracias a la intervención antrópica, que tuvo que realizarse para revertir su degradación producto de la alteración de su dinámica natural. En el área de estudio, la creciente construcción en densidad y altura sobre la línea de costa, así como el desarrollo de la forestación sobre los campos de médanos, han ocasionado que el ecosistema esté bajo una continua alteración, con la consecuente disminución y pérdida de los servicios de regulación evaluados. En aquellas zonas más afectadas por la intervención humana, se ha evidenciado que la protección de las playas favoreció una recuperación más rápida de ciertas playas.

En resumen, de la evaluación de los servicios de regulación en las playas del área de estudio a partir del análisis de las funciones mediante indicadores, puede realizarse un análisis espacial. Las playas del centro del área son las que se encuentran en mejor estado, mientras que las del Norte poseen una peor situación. En éstas últimas, la intervención antrópica ha influido más en los resultados. No obstante, si se analizan los balances sedimentarios particularmente, tanto HR como Monolito son las que poseen mejores valores. Ello se relaciona con la cercanía a las escolleras y espigones que actúan como barrera de contención de la arena, ya que se evidencia una tendencia de recuperación e incremento de su área de playa. Pero como se ha mencionado a lo largo del trabajo, la construcción de obras de defensa se realiza para sustituir en cierta medida, el servicio ecosistémico natural ausente, con lo cual, se evidencia una pérdida o degradación del mismo ocasionado en el área de estudio por la intervención antrópica. Las playas del centro del área de estudio (Coco Loco y Frontera Sur) son las más naturales y las más estables. Chalets, por su parte, si bien posee un estado moderado del servicio, es la que posee los valores más desfavorables en el balance sedimentario. Estas playas son las que necesitan planes de manejo y conservación, que apunten a mantener el flujo de los servicios ecosistémicos permitiendo que las funciones y procesos continúen desarrollándose en forma natural.

\section{Conclusiones}

El estudio de los ecosistemas costeros desde la perspectiva de los servicios y funciones ecosistémicas ha sido de gran utilidad para identificar las problemáticas asociadas en el área de estudio. Los resultados obtenidos podrán emplearse como base para establecer posibles estrategias de gestión. La urbanización sobre el frente costero y la presencia de eventos extremos han impactado de forma negativa sobre el ecosistema y los servicios evaluados. Se ha observado una disminución de los servicios de regulación morfo-sedimentaria y de perturbaciones naturales en las playas, evidenciándose la necesidad de mantener el flujo de los servicios ecosistémicos con el fin de permitir que las funciones y procesos continúen desarrollándose en forma natural.

El área de estudio presenta un estado variado de los servicios debido fundamentalmente, a la variabilidad espacial del funcionamiento del ecosistema. En aquellas playas menos antropizadas, se observó un mejor estado de los servicios, dado que los procesos naturales y estructuras funcionaron equilibradamente. Sin embargo, en aquellas playas más urbanizadas (HR y Monolito) se registró un estado regular debido al impacto de la intervención humana en las mismas. Ejemplo de ello es que el balance sedimentario arrojó resultados positivos, debido principalmente a la presencia de defensas costeras cercanas. Éstas fueron construidas para reemplazar el funcionamiento natural de la playa, el cual había sido alterado por la urbanización y forestación sobre el frente costero y balnearios, y con ello lograr la restauración y continuidad del equilibrio natural. 
Es decir, las obras de defensa se construyeron una vez que el ecosistema fue degradado de tal forma, que fue necesario intervenir para mantener el flujo del servicio.

El buen estado de las playas y sus servicios son de crucial importancia en ciudades cuya economía se basa en el turismo de sol y playa, ya que este tipo de turismo valoriza las condiciones naturales y por ello depende del buen estado de la playa para su desarrollo. Se considera que, de continuar el crecimiento de la urbanización y la actividad turística en el área de estudio, sin la elaboración de un plan de manejo costero, podría acentuarse la tendencia negativa de los servicios evaluados.

Identificar el estado de los servicios ecosistémicos constituye una gran herramienta de gestión para los tomadores de decisiones. Por una parte, permite mediante indicadores, evaluar y monitorear el ecosistema y sus servicios de regulación y por otro, porque de forma sencilla permite ordenar y jerarquizar las zonas de acuerdo a su estado, funciones afectadas y con ello, diseñar estrategias de manejo necesarias para cada una. En aquellas zonas más naturales deben establecerse acciones de preservación para mantener el flujo de servicios, mientras que, en las áreas más críticas, son necesarias acciones de mitigación que permitan incrementar el flujo de servicio.

Este trabajo constituye uno de los primeros aportes al conocimiento sobre el estado de los servicios de regulación en las costas de la provincia de Buenos Aires. La continuidad de esta línea de investigación y monitoreo del área resulta de gran importancia a la hora de gestionar de manera sustentable el ecosistema costero y sus usos y actividades. Más aún, teniendo en cuenta que la principal actividad del área depende directamente del buen estado de conservación y manejo del mismo.

\section{Bibliografía}

ALEXANDRAKIS, G., MANASAKIS, C. \& KAMPANIS, N.A. Valuating the effects of beach erosion to tourism revenue. A management perspective. Ocean \& Coastal Management, 2015, N 111, p. 1-11.

ANFUSO, G., BELLO, E., BENAVENTE, J., NACHITE, D. \& MACIAS, A. Características morfológicas y variabilidad volumétrica de las playas del litoral entre Ceuta y Cabo Negro (Marruecos). Ciencias Marinas, 2006, $N^{\circ} 32$, p. 579-588.

BARBIER, E.B. A spatial model of coastal ecosystem services. Ecological Economics, 2012, $\mathrm{N}^{\circ} 78$, p. 70-79.

BARBIER, E.B. Valuing the storm protection service of estuarine and coastal ecosystems. Ecosystem Services, 2015, No 11, p. 32-38.

BARBIER, E.B., HACKER, S.D., KENNEDY, C., KOCH, E.W., STIER, A.C. \& SILLIMAN, B. R. The value of estuarine and coastal ecosystem services. Ecological Monographs, 2011, N 81, p. 169-193.

BARRAGÁN, J.M. \& BORJA, F. Evaluación de los tipos de ecosistemas operativos: Litorales. En MONTES, C. (Coordinador). Evaluación de los Ecosistemas del Milenio en España. Madrid: UCAUH-UM, 2011, p. 673-739. 
BARRAGÁN, J.M. \& CHICA, J.A. Evaluación de los ecosistemas litorales del milenio de España: una herramienta para la sostenibilidad de la zona costera. Revista EUBACTERIA, 2013, № 31, p. 9-14.

BÉRTOLA, G.R. 21 years of morphological modifications in on urbanized beach (Playa Grande, Mar del Plata, Argentina). Thalassas, 2001, №17, p. 21-36.

BÉRTOLA, G.R., MERLOTTO, A., CORTIZO, L.C. \& ISLA, F.I. Playas de bolsillo en Mar chiquita provincia de Buenos Aires. Revista de la Asociación Geológica Argentina, 2013, Nº 70, p. 267-278.

BRANDER, L.M., FLORAX, R.J.G.M. \& VERMAAT, J.E. The empirics of wetland valuation: A comprehensive summary and a meta-analysis of the literature. Environmental and Resource Economics, 2006, $\mathrm{N}^{\circ} 33$, p. 223-250.

BRENNER, J., JIMÉNEZ, J. A., SARDÁ, R. \& GAROLA, A. An assessment of the non-market value of the ecosystem services provided by the Catalan coastal zone, Spain. Ocean \& Coastal Management, 2010, $N^{\circ} 53$, p. 27-38.

BRUGUERAS, V. Miramar. Compendio Histórico. Mar del Plata: Editorial Martín, 2004.

BURKHARD, B., KROLL, F., NEDKOV, S. \& MÜLLER, F. Mapping ecosystem service supply, demand and budgets. Ecological Indicators, 2012, N²1, p. 17-29.

CHRISTIE, M., REMOUNDOU, K., SIWICKA, E. \& WAINWRIGHT, W. Valuing marine and coastal ecosystem service benefits: Case study of St Vincent and the Grenadines' proposed marine protected areas. Ecosystem Services, 2015, No 11, p. 115-127.

CODIGNOTTO, J.O., DRAGANI, W.C., MARTIN, P.B., SIMIONATO, C.G., MEDINA, R.A. \& ALONSO, G. Wind-wave climate change and increasing erosion in the outer Río de la Plata, Argentina. Continental Shelf Research, 2012, N³8, p. 110-116.

DADÓN, J.R. El frente urbano costero. En: DADÓN, J.R. (editor). Ciudad, paisaje y turismo, Frentes urbanos costeros. Buenos Aires: GEC-FADU-UBA y Nobuko, 2011, p. 165-194.

DE-GROOT R., ALKEMADE, R., BRAAT R., HEIN, L. \& WILLEMEN, L. Challenges in integrating the concept of ecosystem services and values in landscape planning, management and decision making. Ecological Complexity, 2010, № 7, p. 260-272.

EPANCHIN-NIELL, R., KOUSKY, C., THOMPSON, A. \& WALLS, M. Threatened protection: Sea level rise and coastal protected lands of the eastern United States. Ocean \& Coastal Management, $\mathrm{N}^{\circ}$ 137, 2017, pp. 118-130.

FERREIRA, O. The role of storm groups in the erosion of sandy coasts. Earth Surface Processes and Landforms, 2006, $\mathrm{N}^{\circ}$ 31(8), p. 1058-1060.

FIORE, M.M.E., D'ONOFRIO, E.E., POUSA, J.L., SCHNACK, E.J. \& BÉRTOLA, G.R. Storm surges and coastal impacts at Mar del Plata, Argentina. Continental Shelf Research, 2009, № 29, p. 1643-1649. 
FISHER, B. \& TURNER, K.R. Ecosystem services: classification for valuation. Biological Conservation, 2008, No 141, p. 1167-1169.

FOX, W. \& DAVIS JR, R. Seasonal variation in beach erosion and sedimentation on the Oregon coast. The Geological Society of American Bulletin, 1978, № 89, p. 1541-1549.

HAINES-YOUNG, R. \& POTSCHIN, M. The links between biodiversity, ecosystem services and human well-being. En: Raffaelli, D.G. \& Frid C. (editores). Ecosystem Ecology: A New Synthesis. Cambridge: Cambridge University Press, 2010, p. 110-139.

HARRIS, L., NEL, R., HOLNESS, S. AND SCHOEMAN, D. Quantifying cumulative threats to sandy beach ecosystems: A tool to guide ecosystem-based management beyond coastal reserves. Ocean \& Coastal Management, 2015, №110, p. 12-24.

IGM. Lectura de cartografía. Buenos Aires: Instituto Geográfico Militar, 1976.

INDEC. Censo Nacional de Población y Vivienda 2010. Resultados Generales. Provincia de Buenos Aires", Instituto Nacional de Estadísticas y Censos. https://www.indec.gov.ar/nivel4_default. asp?id_tema_1=2\&id_tema_2=41\&id_tema_3=135 (Fecha de consulta: 14/11/2017).

ISLA, F.I. Disponibilidad de arena para el refulado de las playas de Miramar y Chapadmalal, Argentina. Revista de la Asociación Geológica Argentina, 2003, N 58, p. 311-320.

ISLA, F.I., FARENGA, M.O., CORTIZO, L.C., BÉRTOLA, G.R. \& SERRA, S.B. Dinámica morfosedimentaria de playas de arena y grava de la Barrera Austral: Mar del Sud, Arenas Verdes y Costa Bonita. Revista de la Asociación Argentina de Sedimentología, 1997, № 4, p. 15-24.

LA NOTTE, A., D'AMATO, D., MÄKINEN, H., PARACCHINI, M.L., LIQUETE, C., EGOH, B., GENELETTI, D. \& CROSSMAN, N.D. Ecosystem services classification: A systems ecology perspective of the cascade framework. Ecological Indicators, 2017, № 74, p. 392-402.

LIQUETE, C., ZULIAN, G., DELGADO, I., STIPS, A. \& MAES J. Assessment of coastal protection as an ecosystem service in Europe. Ecological Indicators, 2013, N 30, p. 205-217.

MARCOMINI, S.C. \& LÓPEZ, R.A. Alteración de la dinámica costera por efecto de la explotación de arena de playa, partidos de General Alvarado y Lobería, provincia de Buenos Aires. Revista de la Asociación Argentina de Sedimentología, 1999, N 6, p. 1-18.

MARSHALL, F.E., BANKS, K. \& COOK, G.S. Ecosystem indicators for Southeast Florida beaches. Ecological Indicators, 2014, $\mathrm{N}^{\circ} 44$, p. 81-91.

MAYNARD, S., JAMES, D. \& DAVIDSON, A. The development of an ecosystem services framework for South East Queensland. Environmental Management, 2010, № 45, p. 881-895.

MEA (Millennium Ecosystem Assessment). Ecosystems and human well-being: Synthesis. Washington DC: United Nations, Island Press, 2005. 
MEIXLER, M.S. Assessment of Hurricane Sandy damage and resulting loss in ecosystem services in a coastal-urban setting. Ecosystem Services, 2017, $N^{\circ} 24$, p. 28-46.

MERLOTTO, A. \& BÉRTOLA, G.R. Evolución urbana y su influencia en la erosión costera en el balneario Parque Mar Chiquita, Argentina. Papeles de Geografía, 2008, № 47-48, p. 143-158.

MERLOTTO, A. \& BÉRTOLA, G.R. Coastline evolution at Balneario Parque Mar Chiquita, Argentina. Ciencias Marinas, 2009, Nº 35, p. 271-286.

MERLOTTO, A. \& PICCOLO, M.C. Tendencia climática de Necochea-Quequén (1956-2006)", Argentina. Investigaciones Geográficas, 2009; №50, p. 143-167.

MERLOTTO, A., BÉRTOLA, G.R. \& PICCOLO, M.C. Características del oleaje y vientos del sector costero del partido de Necochea, Buenos Aires, Argentina. Revista Geográfica, 2010, № 147, p. 113-131.

MERLOTTO, A., BERTOLA, G.R. \& PICCOLO, M.C. Seasonal morphodynamic classification of beaches in Necochea municipality, Buenos Aires Province, Argentina. Ciencias Marinas, 2013, No 39(4), p. 331-347.

MORTON, R. A., GIBEAUT, J. C. \& PAINE, J. G. Meso-scale transfer of sand during and after storms: implications for prediction of shoreline movement. Marine Geology, 1995, № 126, p. 161-179.

PERILLO, G.M.E., PÉREZ, D.E., PICCOLO, M.C., PALMA, E.D. \& CUADRADO, D.G. Geomorphologic and physical characteristics of a human impacted estuary: Quequén Grande River Estuary, Argentina. Estuarine, Coastal and Shelf Science, 2005, N 62(1-2), p. 301-312.

POUSA, J., TOSI, L., KRUSE, E., GUARAGLIA, D., BONARDI, M., MAZZOLDI, A., RIZZETTO, F. \& SCHNACK, E. Coastal processes and environmental hazards: the Buenos Aires (Argentina) and Venetian (Italy) littorals. Environmental Geology, 2007, N 51, p. 1307-1316.

SCHLACHER, T., SCHOEMAN, D., DUGAN, J., LASTRA, M., JONES, A, SCAPINI, F. \& MCLACHLAN, A. Sandy beach ecosystems: key features, sampling issues, management challenges and climate change impacts. Marine Ecology, 2008, N²9, p. 70-90.

SCHNACK, E.J., POUSA, J.L. \& ISLA, F.I. Erosive processes on the sandy coastline of Argentina. Vechtaer Studien zur Angewandten Geographie und Regionalwiseenschaft, 1998, N²0, p. 133-136.

SHN. Tablas de marea Año 2016. Puerto Mar del Plata, Servicio de Hidrografía Naval. http://www. hidro.gov.ar/oceanografia/tmareas/RE_Mareas.asp (Fecha de consulta: 18/10/2017).

SPALDING, M., RUFFO, S., LACAMBRA, C., MELIANE, I., HALE, L. SHEPARD, C. \& BECK, M. The role of ecosystems in coastal protection: Adapting to climate change and coastal hazards. Ocean \& Coastal Management, 2014, N 90, p. 50-57. 
TURNER, W., BRANDON, T., BROOKS, M., COSTANZA, R., DA FONSECA, G. \& PORTELA, R. Global conservation of biodiversity and ecosystem services. BioScience, 2007, $N^{\circ} 57$, p. 868-873.

VARISCO, C. El cluster turístico de Miramar. Aportes y Transferencias, 2004, № 8, p. 61-88.

VÁSQUEZ, A.E. Infraestructura verde, servicios ecosistémicos y sus aportes para enfrentar el cambio climático en ciudades: el caso del corredor ribereño del río Mapocho en Santiago de Chile. Revista de geografía Norte Grande, 2016, Nº3, 63-86.

VERÓN, E. \& BARRAGÁN, J.M. Transformación y funcionalización del Socioecosistema Litoral Norte de la provincia de Buenos Aires, Argentina. Revista Universitaria de Geografía, 2015, № 24(2), p. 91-116.

VILES, H. \& SPENCER, T. Coastal problems: geomorphology, ecology and society at the coast. New York: Edward Arnoid, 1995. 
